\title{
Review on Research of the Negative Air lon Concentration Distribution and its Correlation with Meteorological Elements in Mountain Tourist Area
}

\author{
Deng Ling \\ Meteorological Service Center, Hunan Provincial Meteorological Bureau, Changsha, China \\ Email address: \\ 274695394@qq.com \\ To cite this article: \\ Deng Ling. Review on Research of the Negative Air Ion Concentration Distribution and its Correlation with Meteorological Elements in \\ Mountain Tourist Area. Earth Sciences. Vol. 8, No. 1, 2019, pp. 60-68. doi: 10.11648/j.earth.20190801.15
}

Received: September 6, 2018; Accepted: November 5, 2018; Published: March 15, 2019

\begin{abstract}
This paper focuses on the research progress of the negative air ion concentration distribution characteristics and its correlation with meteorological elements in mountain tourist areas for recent decades in China. Many researches showed that the distribution of negative air ion in mountain tourist areas has obvious daily and seasonal variations, and its space changes also have regularity. In a day, the NAI concentration will appear alternately with peaks and troughs. The changes of NAI concentration are the summer, autumn high, winter and spring low, the highest in summer, the lowest in winter. In the middle and low altitude areas, NAI concentration is positively correlated with elevation, and negative correlation in high altitude. The concentration of NAI is related to wind speed, air humidity, air temperature, solar radiation, weather conditions, and other meteorological elements. Most research results show that humidity is the most important meteorological factor affecting NAI concentration. The NAI concentration in mountainous scenic spots is significantly positively correlated with relative humidity and rainfall intensity, positively correlated with wind speed and solar radiation, negatively correlated with air temperature. However, due to different sources of monitoring data, observations difference in time, different sample size and other elements, the conclusions on negative air ion concentration distribution characteristics and its correlation with the meteorological factors are not entirely consistent. Negative air ion monitoring equipment should be gradually standardize to ensure the reliability of monitoring data in the future, while we should take advantage of years of continuous observations to carry the study of correlation between negative air ion and meteorological factors, and this will provide a theoretical basis for negative air ion concentration forecasts.
\end{abstract}

Keywords: Mountain Tourist Areas, Negative Air Ion, Concentration Distribution, Meteorological Element, Correlation

\section{Introduction}

Negative air ions (NAI) are the general names of negatively charged single gas molecules and light ion clusters in the atmosphere, and are conventionally referred to as "negative oxygen ion" in China. Under natural or artificial conditions, air molecules and molecular clusters lose electrons into air positive ions, and free electrons can adsorb on the surrounding molecules or molecular clusters to form air anions [1]. There are many ionizers in the atmosphere. There are mainly three kinds of ionizers in the near-surface gas layer: radioactive materials in the earth's crust, radioactive materials in the atmosphere, and cosmic rays and solar ultraviolet rays. In addition, many physical processes in the atmosphere, such as volcanic eruptions, forest fires, photoelectric effects, waves, waterfalls, lightning, snowstorms, and other forms of discharge, can also ionize air molecules, and forming a high concentration of negative air ions [2]. Air anion has the functions of sterilization, dust reduction, and clean air, and is known as "air vitamin and longevity element" [3]. Air anion is very beneficial to human health, and its concentration level has become an important indicator for evaluating a local air quality. The mountain scenic spot is a regional complex with high ecological value, ornamental value and scientific research value based on the mountain as the main landscape resource and landscape element. In recent years, with the rise of ecotourism, the negative ion of air as an ecological health tourism resource 
has attracted more and more attention. Forest and tourismbased forest ecotourism, forest bathing and other activities have been carried out in succession. In China, the forestry, tourism and meteorological departments have established air anion observing stations in cities and scenic spots respectively. The meteorological department has incorporated air negative ions into the meteorological monitoring system, and some provinces and cities have conducted air anion meteorological services for urban and mountainous scenic spots. The study of negative ions also entered a new stage.

Wu Chucai, Shao Hairong, Ni Jun, Li Chenzhen et al . [26] studied the effects of different ecological environment conditions such as forest and city on NAI concentration. Wang Cenglin, Yao Chengsheng, Song Zengwen, and Tan Dong et al. [7-11] studied the NAI concentration distribution characteristics of mountainous scenic spots such as Mount Huangshan and Yuelu. Zhang Zhipan, Tang Chunyan, Xu Zhaohui, Ji Yukai et al. [12-16] studied the NAI concentration distribution characteristics and their relationship with meteorological factors in mountainous scenic spots such as Mount Tianmu, Jinggang and Qipan. From Cong Jing and $\mathrm{Gu}$ Xiaoli et al. [17-18] studied the distribution of NAI concentrations in urban areas and the establishment of prediction models.

This article reviews the observations of domestic scholars on the NAI concentration in mountainous scenic spots over the past decade, and analyzes the temporal and spatial distribution characteristics of NAI concentration in mountainous landscapes and the general rules of the correlation between meteorological elements and the meteorological departments. To develop NAI concentration forecast for mountain-type scenic spots and provide reference and reference for high-quality extension of meteorological services.

\section{Temporal and Spatial Distribution of NAI Concentration in Mountain Tourist Areas}

The concentration of NAI in the natural environment ranges from dozens to tens of thousands per cubic centimeter, and the lifetime ranges from a few seconds to several thousand seconds [19]. Forests are mostly grown in mountain regions, and there are more radioactive substances in mountain rocks; especially ultraviolet rays and cosmic rays are strong on high mountains; The photoelectric effect occurs when sunlight reaches the branches and leaves of forest plants; loose soil under mountain forests, rocks and soils Radioactive elements easily escape the soil and enter the air [2]; in addition, the flow of water, such as waterfalls and streams, is relatively large in forest environment. The aromatic volatile substances released by plants, such as plant essence, can also promote the Ionization of forest air, which increases the NAI concentration [20]. The data show that the concentration of negative ions in the air is significantly positively correlated with plants. The concentration of NAI in the forest is 80 to 1600 times higher than that in urban areas, and the NAI concentration is highest when the forest coverage rate reaches $35 \%$ to $60 \%$ [2]. Due to high forest cover rate, the NAI concentration of Mountain scenic spots is much higher than urban, and the annual average is several thousand to tens of thousands per cubic centimeter [7-15]. For example, the average annual NAI concentration of Mount Huangshan Songgu scenic spot in Anhui Province is as high as $14,000 \mathrm{~cm}^{-3}$, the highest in the summer reached $35000 . \mathrm{cm}^{-3}$ [7]. Many domestic scholars have shown that the NAI concentration distribution in mountain tourist areas has obvious time variations and a certain spatial distribution pattern.

\subsection{The Characteristics of Time Distribution of NAI Concentration}

\subsubsection{Daily Change Characteristics}

The NAI concentration in Mountain scenic spots has obvious daily variation characteristics, and peaks and valleys appear alternately in a day, but there is a certain difference in the appearance time. Most studies showed that $[3,5,7,15-$ $16,21-25]$, the daily variation curve of NAI concentration was bimodal, the overall trend was high at midnight to early morning, low at noon, and gradually increased after the evening. U-shaped distribution, generally two peaks appear in the morning and night respectively. Wu Chucai et al. [3], Yuan Yaoqing et al. [21], Yang Shangying et al. [24] studied the daily changes of NAI in the forest environment of Nanyue Hengshan, Jiemuxi Nature Reserve, and Forest Park of Qingling North Mountain respectively. It was found that there were two peaks of NAI concentrations in one day, the peak value are $07: 00$ to $09: 00$ and $22: 00$ to 24:00 respectively, and the low peak appears at noon or afternoon. Wang Cenglin [7] and Ji Yukai [16] studied the NAI concentrations in Mount Huangshan and Qipan scenic spots. The daily change is: highest in the morning, decreasing at noon or after noon, and high in the evening; one peak at 08:00 during the day, another peak at 22:00 or 00:00, and the lowest at 16:00 or 17:00. Xie Xueyu [22] analyzed the temporal and spatial changes of NAI concentration in the Zhaichangshan Forest Park. The results showed that the peak NAI concentration appeared at 08:00 during the day, began to decline around 12:00, and was lowest between 14:00 and 16:00, then picked up. Chang Yan et al. [23] analyzed that the daily maximum NAI concentration in the forest Daxing'anling Inner Mongolia occurred at 7:00 and 17:00, and the lowest value appeared at 15:00. Mao Chengzhong et al. [25] analyzed that the daily variation trend of NAI concentration in the core area of the Dalaoling National Forest Park in Hubei was higher during the night than in the daytime, during the high concentration period from midnight to morning, the peak appeared at 04:00, and the low concentration period was around noon to evening, the lowest value appeared at 13:00. Photosynthesis of plants is an important source of negative air ions in forest environments 
[9]. Before and after noon, due to the increase of temperature and the decrease of humidity, the photosynthesis "break" phenomenon appeared, resulting in a decrease in NAI concentration [26]. Solar radiation is also a major factor affecting the concentration of NAI, and as the solar radiation decreases in the afternoon, the concentration of NAI decreases. In addition, airborne pollutants such as particulate matter, sulfur dioxide, and nitrogen oxides, which are generated by human activities, also cause a decrease in the NAI concentration. After the evening, as the number of visitors decreased and pollutant emissions decreased, the NAI concentration gradually increased and reached its maximum value in the morning.

\subsubsection{Seasonal Change Characteristics}

Numerous studies have shown [3, 7-8, 12-13, 21-24, 2729] that the seasonal variation of NAI concentration in mountainous scenic spots is significant, showing the rule of high summer and autumn, low winter and spring, and the highest in summer and lowest in winter. However, the concentration of NAI in spring and autumn is not completely consistent. $\mathrm{Wu}$ Chucai et al. [3] conducted comparative observations of air ions in quarters in the Houshan Elm Garden of the Central South Forestry College. July, November, January, and April are respectively representative months for summer, autumn, winter and spring. Each month observed $9 \mathrm{~d}$, the observation time is 08:00, the weather is clear, static and stable, and the average value was taken. The conclusion is that NAI concentration is the highest in summer, autumn is higher than spring, and winter is the lowest; Yao Chengsheng [8], Yuan Yaoqing et al. [22] and Li Peixue et al. [27] also came to the same conclusion by monitoring and analyzing the NAI concentration in Yuelu Mountain, Jiemuxi National Nature Reserve and Jigong Mountain respectively. Yuan Yaoqing et al. [21] also believed that the concentration of NAI was highest in late summer and early autumn, but NAI concentrations in the late autumn, early spring, and winter were not much different. Late autumn and early spring were transitional periods in which NAI concentration rapidly decreased and increased. Zhang Zhipan [12] conducted the observations of NAI concentration on the characteristic plant community in Mount Tianmu from September 2005 to August 2006, it was divided into autumn (September-November), winter (December-February), spring (March-May) and summer (June-August), 3 sunny days are selected for observation each season and observation is made every hour from 08:00 to 17:00. The results showed that the NAI concentration on the community of Cryptomeria fortunei, Ginkgo biloba, Phoebe sheareri and Phyllostachys pubescens in Mount Tianmu was highest in summer, followed by spring and lowest in winter. Wang Cenglin [7] also reached the same conclusion on the observation of NAI concentration in Huangshan Scenic Area. Han Mingchen et al. [29] found that the concentration of NAI in Beigong National Forest Park in summer was significantly higher than that in spring and autumn, and did not change significantly in spring and autumn. High concentration of NAI in summer, which is related to factors such as relatively clean air, high humidity, good hydrological conditions, lush growth of plants, strong photosynthesis and strong solar ultraviolet radiation in this season. The lower concentration in winter is mainly due to the weak photosynthetic activity, relatively serious air pollution, large wind and sand, and frequently fog and haze [26]. The author thinks that the NAI concentrations in spring and autumn are inconsistent, which is mainly because there is no significant difference between them. In spring and autumn the conditions such as plant photosynthesis, solar UV intensity, air cleanliness, and humidity are similar. Resulting the difference of NAI concentration variations are mainly related to differences in observed years or regions.

\subsection{Spatial Distribution of NAI Concentration}

\subsubsection{Vertical Distribution Features}

The NAI concentration and altitude of the mountain landscape show a certain pattern. Wu Fucheng et al. [9] adopted the AIC1000 air ion measuring instrument produced by American Alphalab Company to measure air negative ions at different altitudes at Mount Yuelu (50-300m above sea level) from October in 2003 to September in 2004. Northeast slope (Non-core scenic spot) is divided into 6 measuring points according to the altitude, and the height difference of each measuring point is $50 \mathrm{~m}$. The southeastern slope (core scenic spot) selects the main tourist attractions as measuring points, and a total of 11 monitoring points are set. The study found that with the increase of altitude, the concentration of negative air ions in the northeast slope and the southeast slope gradually increased, reaching the maximum at an altitude of about $150 \mathrm{~m}$, that is, in the low altitude area (50 $150 \mathrm{~m})$, NAI concentration and elevation showed a certain positive correlation . Afterwards, the NAI concentration on both slopes gradually decreased and reached the lowest point on the top of the mountain. This is mainly due to the fact that the foothills are close to the main line of traffic and the population is relatively dense. The smoke and dust caused by human activities increase the probability (or rate) of positive and negative ions annihilating each other, which reduces the concentration of negative ions. The middle of the mountain is shielded by the foothills and the top of the mountain. The role of smoke through the foothills and mountain tops at the tree's absorption and its settlement, its number continued to decrease; coupled with the impact of human activities gradually become smaller, so the air negative ion concentration gradually increased [9]. Ji Yukai etc. [16] studied the air negative ion concentration in Mount Qipan (100-266m above sea level) and reached the same conclusion. That is, in the observed low altitude area (100$220 \mathrm{~m}$ ), the NAI concentration increases with the increase of altitude. Xu Zhaohui [15] in order to study the relationship between the concentration of NAI and altitude, special selection of depressions in the Tiantangzhai Scenic Area as an anion of air anion to eliminate the negative impact of the underlying surface of the forest, water and other negative ions. The study found that NAI concentration was positively 
correlated with elevation in mid-high altitude areas (630$1600 \mathrm{~m}$ ), and negatively correlated with elevations in high altitude areas (1600-1780 m). Wang Shunli etc [30] used the TC.201A intelligent portable air anion detector to locate 7 mountainous terrains such as Mount Xiaolong, Qilian, Lianhua and Taizi in the confluence of Loess Plateau, Qinghai-Tibet Plateau and Mengxin Plateau. In the forest area (elevation 550-5805m), 45 semi-positioning observation points and more than 100 auxiliary observation points are arranged. From July 2008 to August 2009, NAI concentration monitoring in different seasons and different stands is conducted. The correlation between NAI concentration and altitude was also analyzed, and it was also concluded there was a certain negative correlation between NAI concentration and altitude in the high altitude area (1260$3370 \mathrm{~m})$. NAI concentration is closely related to changes in ecological factors, such as vegetation, hydrology and meteorological factors. When these factors are similar, the altitude increases, the air density decreases, and the NAI concentration should decrease accordingly. However, as the altitude increases, the solar radiation increases, the molecular kinetic energy increases, and ion concentration increases. For every 100 meters above sea level, the intensity of solar UV increases by $3 \%$ to $4 \%$. Cosmic rays begin to slowly increase with increasing altitude, and after a constant increase of about $2000 \mathrm{~m}$, it reaches a certain altitude and remains basically constant. In addition, the concentration of NAI is also affected by the atmospheric vertical decline in temperature $\left(0.6^{\circ} \mathrm{C} / 100 \mathrm{~m}\right)$ [15].

\subsubsection{Distribution Characteristics of NAI Concentration Under Different Vegetation Conditions}

The vegetation has a great influence on the concentration of NAI. In different tree species, the concentration of NAI is significantly different. In general, trees with small acicular equiangular radius have the function of "tip discharge", and generate electric charges to ionize the air, thus increasing the concentration of NAI in coniferous forest [2]. In late May 1996, Wu Chucai and others [3] conducted the parallel observation of NAI concentrations on 18 tree species such as Chinese fir, Chinese Liriodendron chinensis, pinus massoniana and so on at tree garden classification area in Hunan Nanyue (altitude 300-500m, subtropical humid monsoon climate zone). The results showed that the average of negative ions in coniferous stands was $1507 . \mathrm{cm}^{-3}$, and broadleaf species was $1161 . \mathrm{cm}^{-3}$. Conifer forest species had higher NAI concentrations than broadleaf species. Ji Yukai et al. [16] chose to observe the average concentration of NAI in 13 species of typical forests in Mount Qipan (100-260 m above sea level, which belongs to the warm temperate zone and semi-humid monsoon climate) in July and August of 2006, and reached the same conclusion. However, Zheng Wenjun et al. [32] conducted comparison of NAI concentration levels in five different types of plant community plots of Guilin Forest Park from May 19 to May 21 in 2008, showing that broad-leaved forests $>$ coniferous forests $>$ mixed needle-width forests $>$ irrigation from $\geq$ turf grass. The reason why the study showed that the concentration of NAI in broad-leaved forests is greater than that of coniferous forests, may be due to different degree of forest density, air circulation conditions and wind speed affect the negative ion level. The negative ions produced by the canopy layer of succulent forest are easily diffused downward by the turbulent flow of the air; the negative ions generated by the photovoltaic effect of the canopy are slowly diffused downward by the canopy. Although the negative ion concentration in canopy layer of dense forest may be higher than that of sparse forest, but under the forest, on the contrary, the content of air negative ions in dense forest is lower than that of the open forest. Xu Zhaohui [15] compared the observations of the isolated forest, sparse forest and dense forest of Pinus taiwanensis in Mount Huangshan Scenic Area between August 2001 and May 2004 with bare land. The sparse forest has the highest contribution rate to air anion, followed by the jungle, and the isolated wood is smaller than the sparse forest and the dense forest. $\mathrm{Xu}$ Zhaohui [15] choose Tiantangzhai National Forest Park (main peak elevation of $1729 \mathrm{~m}$ ) and Kuniujiang Forest Park (master at an altitude $1727 \mathrm{~m}$ ) as research site. DLY-type forest atmospheric ion measurement instrument was used to monitor NAI concentrations under different tree species and under forest and open spaces in May 2004. The study found that the anion density under the forest is significantly higher than it in the dense forest than that of open space, indicating that vegetation played an important role in promoting the production of negative ions, in winter, the contributing rate for the anion of conifer is $49.07 \%$, the broadleaf is $12.49 \% .5$ species of Tiantangzhai National Forest Park among the tree species, eucalyptus, poplar, eucalyptus, chestnut, pine, and bamboo, the highest NAI concentrations are chestnut, eucalyptus and bamboo, the lowest are pine. This is mainly due to the small number of pine trees in various vegetation in Tiantangzhai. There is no population advantage, and the photosynthesis of the needles is not strong, while other tree species have strong photosynthesis and are widely distributed, which can promote the formation of negative ions. There are six common tree species in Kuniujiang forest park, Liriodendron chinense, ginkgo biloba, fragrant fruit tree, banyan tree, eucalyptus forest, bamboo forest. Among these tree species, NAI content is higher in bamboo forest, fragrant fruit tree, and eucalyptus tree. By DLY-5G air ion meter, Zhang Zhipan et al. [12]conducted field observations quarterly (each season choose three sunny day, 08:00-17:00 observation once every 1h)of NAI concentrations on 4 distinct plant communities such as Cryptomeria fortunei, Ginkgo biloba, Phoebe sheareri, and Phyllostachys pubescens in Mount Tianmu (1506 m main elevation, subtropical transition characteristics of the central subtropical zone). Results showed that the average annual NAI concentration for selected plant communities was in the following order: Cryptomeria fortunei (1331 particle $\left.\cdot \mathrm{cm}^{-3}\right)>$ Phoebe sheareri $\left(1223\right.$ particle $\left.\cdot \mathrm{cm}^{-3}\right)>$ Ginkgo biloba $\left(960\right.$ particle $\left.\cdot \mathrm{cm}^{-3}\right)>$ Phyllostachys pubescens (701 particle $\left.\cdot \mathrm{cm}^{-3}\right)$. Wu Jiyou et al. [32] studied the negative ion levels in the air around eight 
garden species and found that the NAI concentrations were significantly different, and the order was Shenshui> Podocarpus $>$ Ledong Unisexual Magnolia $>$ Magnolia $>$ Southern Magnolia $>$ Golden Leaf Mimosa $>$ Lechang Magnolia $>$ Chinese Liriodendron chinensis. In addition, from the planting mode, the NAI concentration shows a mixture of arbor and shrub-grass forest $>$ Qiao irrigation mixed forest $>$ single-layer arbor $>$ Joe Grass $\operatorname{mix}>$ shrub mix $>$ monolayer shrub $>$ single grass cover feature [33] .

\section{Correlation Between NAI Concentration Changes and Meteorological Elements in Mountainous Scenic Spots}

The generation of air negative ions is closely related to the meteorological conditions. From the analysis of the formation reasons, weather phenomena such as heavy rain, lightning, and ultraviolet (solar radiation) are favorable to various ionization reactions in the air and release electrons. In addition, the presence or absence of negative ions in the air is related to the cleanliness of the air, and the degree of cleanliness of the air is closely related to the weather, especially wind speed, wind direction, and fog [34]. Studies have shown that NAI concentration has a certain correlation with weather factors such as wind speed, wind direction, air temperature, relative humidity, visibility, solar radiation, and weather conditions, among which humidity is the most important meteorological factor affecting NAI concentration.

\subsection{Correlation Between NAI Concentration and Wind Speed}

The size of the wind speed is a key factor in determining the cleanliness of the air, and air cleanliness is closely related to the survival of negative air ions. In addition, from the microscopic analysis, during the formation of air negative ions, it is necessary to obtain energy from the molecular state to the ion state. Under strong wind conditions, the opportunity for molecules to obtain energy increases, and the process of changing the molecular state to the ion state is accelerated. Therefore, wind is one of the meteorological factors that affect the change of NAI concentration. There is little research on the correlation between wind speed and NAI concentration in mountainous scenic spots in China. Si Tingting et al. [14] monitored the NAI concentration and meteorological elements in Diaoluoshan (an altitude of 944 $\mathrm{m}$, tropical marine monsoon climate), and found that the relationship between NAI concentration and wind speed of tropical rainforest is not significant under rainy or non-rainy conditions. Many scholars [34, 36-39] studied the effect of wind on air negative ions in urban areas, wetlands and seashores. For example, Ye Caihua et al. [34] conducted stepwise regression analysis on daily observation data of NAI concentration in Beijing and corresponding meteorological elements, it was found that there was a significant positive correlation between NAI concentration in the air and daily average wind speed. Wang Xiaoyun et al. [36] conducted simultaneous observations of hourly negative ion concentration and meteorological elements for 11 consecutive days from March 4 to March 14, 2001, 24 hours a day, and also found that the daily variation of NAI concentration was positively correlated with the daily change of wind speed near the surface. When the wind speed increases near the ground $(1.5 \mathrm{~m})$, the NAI concentration increases simultaneously. Qi Bing et al. [37] used the observation data of negative ions in the Mount Mantou and Xixi wetlands in Hangzhou during 2008 to 2010 . Results showed that the NAI concentration was positively correlated with the wind speed. In March 1989 to February 1990, the concentration of air negative ions, wind direction and wind speed were observed regularly and at fixed points in Gulangyu, Xiamen by Wang Jinqiu et al. [38], and it showed that the greater the wind speed, the higher the concentration of seashore NAI.

\subsection{Correlation of NAI Concentration and Relative Humidity}

According to the theory of the Joint Commission on Atmospheric Research of the International Union of Geodesy and Geophysics, the formula for air anions is $\mathrm{O}_{2}{ }^{-}\left(\mathrm{H}_{2} \mathrm{O}\right) n$, or $\mathrm{OH}^{-}\left(\mathrm{H}_{2} \mathrm{O}\right) \mathrm{n}$, or $\mathrm{CO}_{4}^{-}\left(\mathrm{H}_{2} \mathrm{O}\right)_{2}$. It can be inferred that humidity has a greater influence on air negative ion concentration [39]. Huang Shicheng et al. [40] used negative ion observation data and meteorological observation data from cities and forests in Suqian city for one year to use path analysis methods to conclude that the daily mean vapor pressure is the largest direct factor and the largest indirect factor for atmospheric negative ion concentration. Numerous studies $[12,16,21,27,30,41-42]$ have shown that the NAI concentration in mountainous scenic spots is significantly positively correlated with the relative humidity of the air, that is, the NAI concentration increases with relative humidity in the corresponding interval. For example, In Qipan Mountain Scenic Area, NAI concentration is positively correlated with relative humidity at $60 \% \sim 96 \%$, and the correlation coefficient is $\mathrm{R}=0.891$ [16]. In Mount Tianmu Scenic Area, the correlation coefficient between the NAI concentration and relative humidity at $86 \% \sim 96 \%$ is 0.820 [12]. There is a positive correlation between the NAI concentration of Mount Dashu Forest Park and relative humidity at $40 \% \sim 84 \%$, the correlation coefficient is $\mathrm{R}=0.820$ [42], and the correlation coefficients of air relative humidity and NAI concentration in Jiemuxi Nature Reserve and seven forest areas in Gansu province were 0.7919 and 0.6801 respectively. Wang Jimei et al. [43] obtained a simulation of the temperature and humidity under natural conditions, and found that the NAI concentration was positively correlated with relative humidity, and the rate of change of different humidity stages was different. When the humidity is less than $60 \%$, the NAI concentration increases with the increase of humidity, but the increase is small, and when the humidity gradually increases from $60 \%$ to $80 \%$, the NAI concentration increases 
significantly.

\subsection{Correlation Between NAI Concentration and Air Temperature}

Air temperature is also one of the main meteorological factors affecting NAI concentration. Huang Shicheng et al. [40] believe that among many meteorological factors, the daily average temperature has a direct effect on the concentration of negative ions in the forest area only after the average vapor pressure. Studies $[12,16,30,41]$ have shown that, within the corresponding temperature range, there is a significant negative correlation between NAI concentration and ambient temperature in mountainous scenic spots, and NAI concentration decreases with increasing temperature. In the range of $23 \sim 27^{\circ} \mathrm{C}$, the correlation coefficient of NAI concentration and air temperature in Mount Tianmu Nature Reserve is $\mathrm{R}=0.676$; in the range of $18 \sim 30^{\circ} \mathrm{C}$, the correlation coefficient of NAI concentration and temperature in Qipanshan Scenic Area is $\mathrm{R}=0.728$; Within the range of $5 \sim 20^{\circ} \mathrm{C}$, the NAI concentration and temperature in the 7 large forest areas of Gansu also showed extremely significant negative correlation. The correlation coefficient between NAI concentration and air temperature of Zhunian Waterfall and Niujiaolong of Shennong Valley National Forest Park reached -0.775 and -0.812 respectively. The concentration of NAI decreased with increasing ambient temperature, and the main reason was that the temperature increased and the diffusion of pollutants decreased. The pollutants adsorbed a large amount of air negative ions during the diffusion process, thus reducing the concentration of NAI. However, some scholars [27, 34-36, 41] have reached the contrary conclusion that NAI concentration is positively correlated with temperature, that is, NAI concentration increases with increasing temperature. For example, Li Peixue et al. [27] used the average value of air negative ion concentration in seven different habitats in Jigong Mountain from June 2007 to May 2010 to conduct correlation analysis with temperature, and found that the NAI concentration was significant positively related with the air temperature within $0-25^{\circ} \mathrm{C}$, the correlation coefficient of 0.9645 . Wang Jimei et al. [43] simulated the temperature and humidity in the natural environment. The study showed that the NAI concentration was positively correlated with air temperature in the range of $5-40^{\circ} \mathrm{C}$ under the condition of humidity $35 \%$, but there was no significant influence of humidity. This is because an increase in temperature can accelerate the thermal motion of molecules or atoms, increase the probability of collision and ionization, and increase the ionization energy of oxygen. When the temperature rises, the average kinetic energy of molecules or atoms increases, and the ability of oxygen to be ionized increases. Negative oxygen ions increase, negative ion concentration increases. In addition, the saturated vapor pressure of water increases exponentially with increasing temperature. When the relative humidity remains constant, the water molecule content per unit volume also increases exponentially, so the concentration of negative air ions increases . Another research [16] holds that the relationship between air negative ions and temperature is not significant.

\subsection{Correlation Between NAI Concentration and Solar Radiation}

Solar radiation provides energy for various ionization reactions in the air. Meanwhile, solar radiation is strong, Photosynthesis of forests is strong, and plants release more oxygen into the atmosphere through photosynthesis. Oxygen molecules have a strong ability to absorb free electrons in the air, so the concentration of negative ions in the air is higher with the the increase of solar radiation. Xu Zhaohui [15] based on the analysis of daily observation data of NAI concentration in Tiantangzhai Scenic Spot, selects the air negative ion concentration of the sunny and cloudy weather types and the corresponding meteorological factors to perform single factor correlation analysis. The results showed that the NAI concentration and the daily total amount of solar radiation showed a very significant linear positive correlation, and the daily total amount of solar radiation increased by $1 \mathrm{MJ} \cdot \mathrm{m}^{-2}$, and the NAI concentration increased by $30 \cdot \mathrm{cm}^{-3}$. Ye Caihua et al. [34] believed that the NAI concentration is directly proportional to the direct solar radiation; Wang Xiaoyun et al. [36] also found that the NAI concentration changed in the same phase as the solar ultraviolet radiation intensity, that is, the solar ultraviolet changed in radiation intensity is large, the NAI concentration was high, and the solar ultraviolet radiation intensity was small, and the NAI concentration was low. Yuan Yuqing et al. [19] calculated that the correlation coefficient between light intensity and NAI concentration in Jiemuxi nature reserve was 0.6083 .

\subsection{Effect of Weather on NAI Concentration}

The NAI concentration also varies with different weather conditions. Studies have shown that after rainfall, the NAI concentration is significantly higher than that of cloudy and sunny days, while the NAI concentrations of sunny days are slightly higher than that of cloudy days $[9,13,16,21,25,27$, 38]. For example, $\mathrm{Wu}$ fucheng et al. [9] found that NAI concentration increased after raining in Mount Yuelu of changsha, and the air quality generally increased by one grade. In sunny day, NAI concentration was slightly higher than that in cloudy day, with little change in air quality. Tang Chunyan et al. [13] found that the concentration of NAI in Mount Jinggang increased significantly after showers, intermittent drizzles, and thunderstorms; Ji Yukai et al. [16] showed that the NAI concentrations of five different sites in Qipan Mountain reached the highest value after raining. The NAI concentration in each season after raining was significantly higher than that before rainfall, and the winter, spring, summer and autumn concentrations increased by $20 \%, 16.03 \%, 14.05 \%$, and $14.68 \%$, respectively. The NAI concentrations in sunny winter, spring, summer, and autumn were respectively $36,30,60$ and $45 \mathrm{~cm}^{-3}$ higher than cloudy day [21]. Mao Chengzhong et al. [25] found that the 
concentration of NAI in the core area of the Dalaoling National Forest Park in Hubei was higher in sunny days and thunderstorms than in cloudy days. Li Peixue [27] investigated that in different weather conditions in Mount jigong, the concentration of NAI was successively higher than that of sunny days rather than cloudy ones. After the rain, the increase in the concentration of negative air ions is due to the fact that after the rain, a large amount of dust in the air collides with the rain and splashes onto the ground. The air is more refreshing and prolongs the life of the negative ions in the air. In addition, rain drops and splashes break the water droplets. It is easy to form charged water molecules and increase the rate of negative ions in the air. Therefore, the NAI concentration in the weather after rain is significantly higher than that in sunny and cloudy days, and the NAI concentration will increase significantly on the first and second days after the occurrence of thunderstorm or rain above the medium rain [33]. Sunny days are higher than cloudy days due to the strong sunshine on sunny days. Abundant ultraviolet rays contribute to the production of negative air ions, and the photosynthesis of plants is also strong. Si tingting et al. [43] analyzed the NAI concentration and meteorological elements in the Diaoluo Mountain (elevation $744 \mathrm{~m}$, tropical marine monsoon climate), and analyzed that there was a significant positive correlation between NAI concentration and rainfall intensity in tropical rain forests, and NAI concentration variation and rainfall. There was a significant positive correlation between the change in rainfall intensity when there was a change in intensity, and there was no significant difference between the negative ion concentration and the non-rainfall conditions when the rainfall intensity was small (less than $2 \mathrm{~mm}$ within $15 \mathrm{~min})$.

\section{Conclusions}

Mountainous scenic spots have obvious advantages in air anion resources, and air anion tourist resources, as an emerging eco-tourism tourism resource with development prospects in scenic spots, is of great significance to tourism. Strengthening the study of NAI concentration distribution can guide people to make better use of air negative ion resources to strengthen their health. In the past decade, many domestic scholars have initially observed the distribution characteristics of NAI concentration in different mountainous scenic spots and their relationship with meteorological elements. Studies have shown that the air negative ion concentration in mountainous scenic spots has a significant seasonal change, vertical change is also very obvious, and there are significant differences under different vegetation conditions. In a day, the NAI concentration will appear alternately with peaks and troughs. The overall trend is high from midnight to early morning and low at noon, and after the evening, it gradually rises, showing a U-shaped distribution. Generally, the two peaks appear in the morning and at night. The changes of NAI concentration are the summer, autumn high, winter and spring low, the highest in summer, the lowest in winter, the difference is not obvious in spring and summer. In the middle and low altitude areas, NAI concentration is positively correlated with elevation, and negative correlation in high altitude. It is believed that the concentration of NAI in coniferous forests is greater than that in broad-leaved forests, and the concentration of NAI in forest lands is greater than that in empty lands. The concentration of NAI is related to wind speed, air humidity, air temperature, solar radiation, weather conditions, and other meteorological elements. Most research results show that humidity is the most important meteorological factor affecting NAI concentration. The NAI concentration in mountainous scenic spots is significantly positively correlated with relative humidity and rainfall intensity, positively correlated with wind speed and solar radiation, negatively correlated with air temperature. After rainfall, NAI concentration significantly increased, and the size of NAI concentration was in the following order: sunny days after the rain $>$ sunny days $>$ cloudy days. However, some studies have also found that NAI concentration is negatively correlated with wind speed, positively correlated with air temperature, or has no significant correlation. This shows that the consistency between different research results is relatively lacking. This shows that the consistency between different research results is relatively lacking. The reason for this may be related to the different methods of obtaining monitoring data and statistical analysis methods used by different researchers, and also to the meteorological elements in different environments. The complexity of the interactions, the geographical location of the study area, the climate background, and the underlying surface differences are related. In summary, the author believes that the performance of air negative ion self-monitoring instruments should be continuously improved, and the calibration and comparison of instrumentation equipment should be standardized to ensure the reliability of the monitoring data, and an easy-to-carry observation instrument should be developed to better adapt to various mountain-type scenic spots. Long-term synchronous location monitoring of NAI concentration in different mountainous scenic areas should be carried out, and continuous observation data for many years should be used to study the correlation between NAI concentration distribution and meteorological elements. Further studies on the mechanism of air negative ions generation should be strengthened to provide a theoretical basis for the predication of NAI concentration.

\section{References}

[1] Lin Yuchun. The environmental ecological effects of air oxygen. Ecologic Science. Vol.19, No. 2, 2000, pp. 87-90. (in Chinese).

[2] Shao Hirong, He Qingtang. Forest and air anion . World Forestry Research. Vol. 37, No. 5, 2001, pp. 75-81. (in Chinese).

[3] Wu Chucai, Zheng Qunming, Zhong Linsheng. A Study of the Aero-Anion concentration in forest recreation area. Forestry Science. Vol. 37, No. 5, 2001, pp. 75-81. (in Chinese). 
[4] Ni Jun, Xu Qiong, Shil Dengrong, et al. The correlative study of air anions in city greenbelt: A case of the parks in Shanghai. Chinese Urban Forestry, Vol. 2, No. 3, 2004, pp. 30-33. (in Chinese).

[5] Zhao Xiongwei, Li Chunyou, Ge Jingru, et al. Progress of aero anion in forest environment . Journal of Northwest Forestry University. Vol. 22, No. 2, 2007, pp. 57-61. (in Chinese).

[6] Li Chenzhen, Gan Dexin, Chen Xiaoying. Different ecological conditions on the concentration aero anion research . Modern Agricultural Science. Vol. 16, No. 5, 2009, pp. 174-176. (in Chinese).

[7] Wang Cengling. Study on the cause of negative ion tourism resources distribution and development in Mount Huangshan scenic area. Hefei: Anhui Agricultural University, 2003, pp. 1-57.(in Chinese).

[8] Yao Chengsheng. Study on the distribution and utilization of air negative ions in Yuelu Mountain. Changsha: Changsha Teacher-training University, 2005, pp. 1-45. (in Chinese).

[9] Wu FuCheng, Yao Chengsheng, Guo Jianping, et al. Distribution of negative air ions and its relation to air quality of the Yuelu Mountain . Acta Scientiae Circumstantiae, Vol. 26, No. 10, 2006, pp. 1737-1744. (in Chinese).

[10] Song Zengwen, Xian Baohui, Zhong Linsheng. Different ecological conditions on the concentration aero anion research . Modern Agricultural Science. Vol. 28, No. 3, 2008, pp. 211-215. (in Chinese).

[11] Tan Dong, Zhang Xiangsheng, Yang Juan. A primary exploration on distribution and the variation ofnegative oxygen ion concentration in ChaShanZhuHai . Environment and Ecology in the Three Gorges. Vol. 186, No. 3, 2010, pp. 26-28. (in Chinese).

[12] Zhang Zhipan, Yu Yiwu, Zhang Mingru et al. Negative air ion concentration and environmental factors for Mount Tianmu. Journal of Zhejiang Forestry College, Vol. 25, No. 4, 2008, pp. 481-485. (in Chinese).

[13] Tang Chunyan, Cai Zhe, Xiao An , et al. Concentration characteristics of air negative Ion and Its relations with meteorological conditions in Jinggang Mountain. Journal of Anhui Agri. Sci, Vol. 39, No. 1, 2011, pp. 495-496. (in Chinese).

[14] Si Tingting, Luo Yanju, Zhao Zhizhong, et al. Relationship between negative air Ion concentration and meteorological elements in Diaoluoshan tropical rainforest. Resources Science, Vol. 36, No. 4, 2014, pp. 0788-0792. (in Chinese).

[15] Xu Zhaohui. The anion resources research in tourism area of Anhui province. Hefei: Anhui Agricultural University , 2004, pp. 1-58. (in Chinese).

[16] Ji Yukai. Study on the distributing and changing low of aeroation in Qipan Moutain scenic spot. Shenyang: Shenyang Agricultural University, 2007, pp. 1-46. (in Chinese).

[17] Cong Jing, Sun Lijuan. Distribution of negative oxygen ion concentration and its forecast model in Dalian City. OurnalL of meteorology and environment, Vol. 26, No. 4, 2010, pp. 4447. (in Chinese).

[18] Gu Xiaoli, Qian Yanzhen, Bao Yuejian, et al. The distribution of oxygen anion concentration, forecasting model in Ningbo and its application in tourism meteorology. Journal of Meteorology and Environment, Journal of Zhejiang Forestry College, Vol. 29, No. 6, 2013, pp. 128-133. (in Chinese).

[19] Laakso L, Hussein T, Aarnio P. etc. Diurnal and annual characteristics of particle mass and number concentrations in urban, rural and Arctic environments in Finland. Atmospheric Environment, Vol. 37, 2003, pp. 2629-2641.

[20] Zeng Shucai, Su Zhiyao, Chen Beiguang. Review on forest negative air ions in China. Journal of Nanjing Forestry University (Natural Sciences Edition), Vol. 30, No. 5, 2006, pp. 107-111. (in Chinese).

[21] Yuan Raoqing, Wen Hong Variation of air anion concentration before and after precipitation in Jiemuxi Nature Reserve. Journal of Anhui Agri . Sci, Vol. 38, No. 17, 2010, pp. 91069109. (in Chinese).

[22] Xie Xueyu, Hu Xijun , Zhu Xuanni, Spation-temporal variation characteristics of negative air ion concertrations in Zhaichangshan Forest Park. Journal of Northwest Forestry University, Vol. 30, No. 5, 2014, pp. 37-41. (in Chinese).

[23] Chang Yan, Wang Qingmin, Zhang Qiuliang, et al. The variation and valuation of anion concentration in the forest Daxinganling Inner Mongolia. Journal of Inner Mongolia Agricultural University, Vol. 31, No. 1, 2010, pp. 83-87. (in Chinese).

[24] Yang Shangying,. Developing aero anion resource in Forest Park of Qingling North Mountain. Resource Development \& Market, Vol. 21, No. 5, 2005, pp. 458-459. (in Chinese).

[25] Mao Chengzhong, Yu Nailian, Du Jiale, et al. Characteristic comparison of negative oxygen ions between typical urban and forest areas. Meteorological Science and Technologyt, Vol. 42, No. 6, 2014, pp. 1083-1089. (in Chinese).

[26] Pan Ruichi. Psychophysiology. Beijing: Advanced education press, 2004.

[27] Li Peixue, Dai Huitang, Yang Huai, et al. Variation of negative air ion and Its relationship with environmental factors in Jigongshan. Journal of Xinyang Normal University Natural Science Edition, Vol. 24, No. 1, 2011, pp. 79-84.

[28] Wang Shujuan, Wang Fang, Guo Jungang, et al. A review: negative air ions and Its influence factors in forest areas. Journal of Inner Mongolia Agricultural University, Vol. 29, No. 1, 2008, pp. 243-247.

[29] Han Mingchen, Ye Bing , Zhang Decheng. Aero-anion concentration in Beigong National Forest Park and ecological value assessment. Journal of West China Forestry Science, Vol. 42, No. 1, 2013, pp. 32-37.

[30] Wang Shunli, Liu Xiande, Jin ming, et al. Distribution characteristics of negative air ions in forest region of Gansu. Ecology and Environmental Sciences, Vol. 19, No. 7, 2010, pp. 1563-1568.

[31] Zheng Wejun, Wang Jinye, Li Haiming. Preliminary Study on Aero-Anion Concentration of Different Vegetation Types in Forestry Recreational Areas. Fujian Forestry Science and Technology, Vol. 36, No. 2, 2009, pp. 98-100.

[32] Wu Jiyou, Cheng Zhenghong, Long Yingzhong, et a1. The variation of aero anion concentration on landscape forest. Journal of Nanjing Forestry University: Natural Sciences Edition, Vol. 27, No. 4, 2003, pp. 78-80. 
[33] Su Shuquan. Study on different stand types influence to air quality. Central South Forest Inventory and Planning, Vol. 21, No. 4, 2002, pp. 47-48.

[34] Ye Caihua, Wang Xiaoyun, Guo Wenli. A preliminary study on the relationship between the concentration of negative ions in air and meteorological conditions. Meteorological Science and Technology, No. 4, 2000, pp. 51-52.

[35] Chen Huan, Zhang Jiaen. Review on factors influencing the concentration distribution of negative air ions. Ecological Science, Vol. 29, No. 2, 2010, pp. 181-185.

[36] Wang Xiaoyun, Ji Chongping, Hufei, et al. Study on relationship between the concentration of air negative ions in the near stratum and meteorological conditions. Proceedings of the scientific symposium on urban meteorological services, 2010, pp. 79-83.

[37] Qi Bing, Du Rongguang, Shao Bijia. Charcterstics of aion variation in Hangzhou. Meteor Disaster Reduction Res, Vol. 34, No. 4, 2011, pp. 68-71.

[38] Wang Jinqiu, Li Xiuzeng, Cai Jinpen. The effect of sea waves on the concentration of negative ions in coastal air. Chinese physiotherapy journal, No. 4, 1991, pp. 157-158.
[39] Zhang Zhipan, Yu Yiwu, Meng Minghao, et al. Progresses of aero anion in tourism environments. Journal of Zhejiang Forestry College, Vol. 26, No. 1, 2006, pp. 103-108.

[40] Huang Shicheng, Xu Chunyang, Zhou Jialing. Path analysis on negative air ion coneentration and the Meteorological environment in urban and forest zones. Meteorological Monthly, Vol. 28, No. 11, 2012, pp. 1417-1422.

[41] Zhang Shuangquan, Tan Yiming, Wu Zhangwen, et al. The relationship between air anion concentration and air temperature and air relative humidity. Journal of Central South of University of Forestry and Techology, Vol. 31, No. 4, 2011, pp. 114-118.

[42] Wei Chaoling, Wang Jingtao, Jiang Yuelin, et al. Air negative charge ion concentration and its relationships with meteorological factors in different ecological functional zones of Hefei City. Chinese Journal of Applied Ecology, Vol. 17, No. 11, 2006, pp. 2158-2162.

[43] Wang Jimei, Ji Zhijiang, Sui Tongbo, et al. Influence of temperature and humidity on negative ion concentration. Research of Environmental Sciences, Vol. 17, No. 2, 2004, pp. 68-70. 